\title{
Experimental Modeling of Monolithic Resistors for Silicon ICS with a Robust Optimizer-Driving Scheme
}

\author{
Philippe Leduc $^{(1,2)}$, Didier Magnon ${ }^{(1)}$, and Fabrice Guitton ${ }^{(2)}$
}

(1) Power Microelectronic Laboratory (L.M.P), Tours University, 16 rue Pierre et Marie Curie, 37071 Tours Cedex 2, ${ }^{(2)}$ Application and System Engineering STMicroelectronics, 16 rue Pierre et Marie Curie, 37071 Tours Cedex 2, FRANCE, E-mail: philippe.leduc@st.com

النوذج المعملي للفقاومل" Monolithic " المستخمة ف Silicon RF IC's للالم الل ومتنن

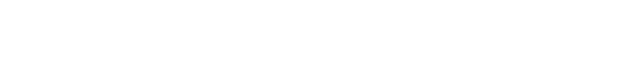

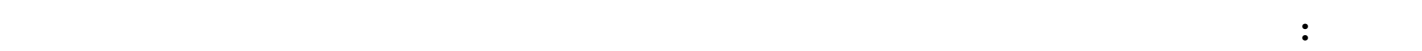

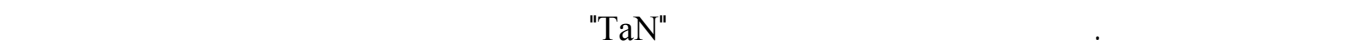
(I- Type )

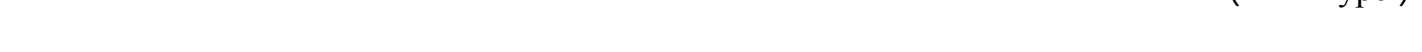

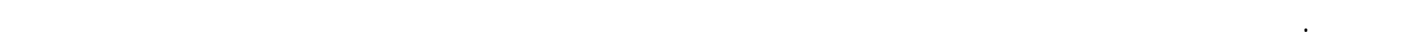
الذالة.

\begin{abstract}
Today, an exhaustive library of models describing the electrical behavior of integrated passive components in the radio-frequency range is essential for the simulation and optimization of complex circuits. In this work, a preliminary study has been done on Tantalum Nitride (TaN) resistors integrated on silicon, and this leads to a single $\pi$-type lumped-element circuit. An efficient extraction technique will be presented to provide a computer-driven optimizer with relevant initial model parameter values (the "guess-timate"). The results show the unicity in most cases of the lumped element determination, which leads to a precise simulation of self-resonant frequencies.
\end{abstract}

KEYWORDS: RF ICs, TaN resistors, Experimental Modeling, Circuit Optimization, Guess-Timate.

\section{Introduction}

$\mathbf{T}$

The current trend in the development of application is to combine silicon integrated circuits and high frequency circuits into a compact module. The impact of such an innovation is huge since it concerns common electronic devices: pagers, mobile phones, global positioning systems, all of which work in the radio-frequency domain. If the integration of passive components directly aims at decreasing circuit size, the electrical performance has also improved limiting interconnection parasitics.

Due to the micrometric dimensions of integrated circuits, electromagnetic interactions of a component with the surrounding environment become non-negligible and lead to non-trivial frequency responses. In order to anticipate design problems and quickly propose a product meeting specification requirements, it is primordial to precisely predict the behavior of a large number of passives as a function of their geometry, in a frequency range as wide as possible (until $6 \mathrm{GHz}$ ). The characterization of passive components requires at microwave frequencies to take into account physical phenomena such as fringing fields, skin effect, eddy currents (Chen and Munoz, 1997)... These phenomena, closely related to the manufacturing process and the component geometry, are 
much too complex to be solved analytically. Today, electromagnetic field-solvers are available and evaluate the field distribution using various numerical methods. To draw up an exhaustive review of all the techniques that have been proposed over the last five decades would be impractical here. Instead, only the techniques that have been commonly implemented in commercial simulators will be mentioned, such as the Finite Difference Time Domain Method (FDTD) (Ciampolini et al., 1996), the Finite Element Method (FEM) (Itoh ,1989), the Method of Moments (MoM) (Harrington, 1968) and the Transmission Line Matrix Method (TLM) (Johns, 1987). A major drawback of three-dimensional (3D) structure simulations is that sophisticated skills in numerical analysis on behalf of the user are required in order to get meaningful results. Furthermore, processing speed and memory capacity available on calculators considerably limit the size of the structures which can be analyzed within a reasonable amount of time (Long and Copeland, 1997), especially if a sufficiently fine array of sampling nodes is chosen to have any hope of an accurate computation. 2D and 2.5D simulators are rapid and allow the study of complex layouts respectively in a planar and mostly planar configuration. Nevertheless, they remain inadequate because they do not completely describe the interaction with the subjacent substrate.

Fruitful attempts have been led to interpolate the measured response with a rational polynomial function (Choi and Swaminathan, 1999). A synthesis technique is then applied to generate a lumped element equivalent circuit. However, this method cannot ensure positive circuit elements to fully correlate with the physical layout. We believe a simplified circuit model based on the insights achieved from measurements greatly simplifies the numerical modeling process. Advanced problems are often best solved by an evolutionary approach whereby a problem with a smaller number of variables is solved first. It also provides the circuit designer, thanks to the direct relation that can be established between a physical phenomenon and its electrical representation, with a deeper understanding of the component behavior. Although electrical models derived in this experimental way cannot be scaled to reflect changes in the layout or fabrication technology, they are still imperative for more advanced models. A scalable model allows the circuit designer the flexibility to tailor the design for a given RF application. Scalability implies that the electrical circuit parameters can be deduced from geometric and technological specifications, and consequently is closely linked to the underlying physics (Yue and Wong, 2000). They can be directly used in the time domain, performing for instance a transient analysis with SPICE, or in the frequency domain, using tools such as Touchstone. The complete model can also be reduced to a compact form or a reflection representation (S-parameter matrix) for a fast optimization of complex RF circuits.

This objective cannot be reached without a robust method to extract the model elements. Zhao et al. (1997) have shown that S-parameters' sensitivities to circuits parameters differ enormously, which means that for one objective function, local minima may very easily upset the minimization process. Moreover, they propose a very interesting optimization method based on an exponential gradient learning algorithm, stemming from neural network research. Once again, such a method needs good expertise in computer science to be fully exploited. This paper aims to put forward a parameter extraction process based on a deep and rigorous analysis of the model elementary cell. Indeed, mathematical relations can be established for model component values to be compatible with the few qualitative measurement configurations obtained. On the one hand, the dimension of the fitting problem, the parameter variation extent and consequently, the problem complexity, are diminished. On the other hand, by providing the optimizer with good initial values, we can reduce the chance of hitting local minima. Choosing the appropriate initial values not only speeds up the minimization process, but also gives rough interpretation of the measured data. Thus, a commercial computer-driven optimizer oriented with explicit optimization rules becomes a powerful investigation tool.

\section{Test Fixture Design}

In order to obtain valuable insights into the embedded resistors, a set of resistors with increasing sizes as well as their testing structures were built onto wafer, as shown in Figure 1. 


\section{EXPERIMENTAL MODELING OF MONOLITHIC RESISTORS}
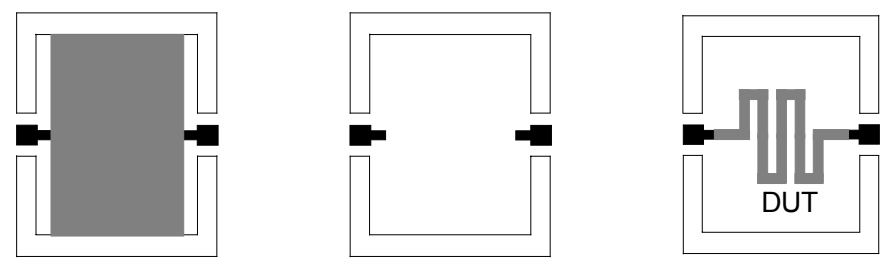

Figure 1. Short, open and load coupons.

Experimental data were collected from the measurements of the two-port S-parameters for each resistor dummy structures. Ceramic Impedance-Standard-Substrate (ISS), which provides the standard short, open, load and thru fixtures (SOLT), was used to calibrate until $6 \mathrm{GHz}$ the general 2-port network analyzer HP-8753E up to PicoProbe coplanar groundsignal-ground probes. A de-embedding procedure is necessary in order to extract the useful signal from the measurement environment. In this way, each Device-Under-Test (DUT) is accompanied by an empty pattern designed to measure the influence of the ground ring necessary to ground the probes for the network analyzer, and a filled pattern to measure the inductive effects of the pads and access lines necessary for connecting the probes to the component. A common compensation technique consists in subtracting the $Y$ parameters of the open pattern to the total $Y$, and then subtracting the $Z$ parameters of the short pattern to the resulting $Z$ matrix. This procedure proved to be inadequate in our case, because of the testing pattern structure. The Three-Step method was preferred (Kolding, 1999): this involves an electrical representation of the different patterns to explicitly express the parallel $\left(Z_{\mathrm{p} 1}, Z_{\mathrm{p} 2}\right)$, serie $\left(Z_{\mathrm{s} 1}, Z_{\mathrm{s} 2}\right)$, and transmission $\left(Z_{\mathrm{open}}\right)$ related parasitics, shown in Figure 2, as functions of the measured scattering parameters.

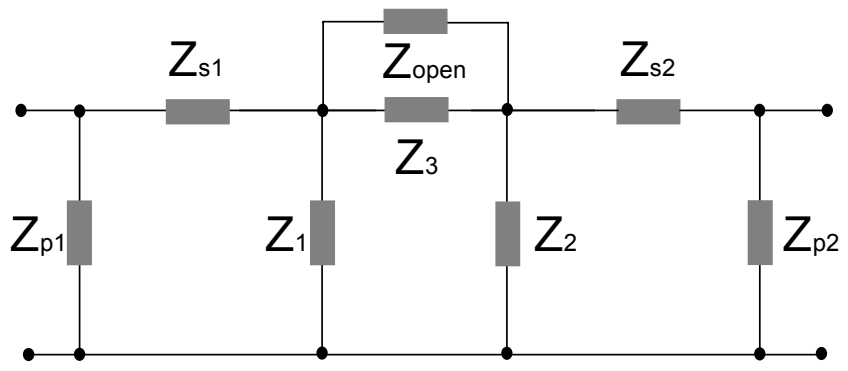

Figure 2. Embedded device modeling.

\section{Equivalent Circuit Model}

A single $\pi$-type lumped-element circuit (as shown in Figure 3) has been used to fit experimental results. This idealized representation, the simplest mentioned in the literature, is a first step to build up a model library since it permits the modeling process set up to be validated. The equivalent circuit is based on our understanding of the integrated resistor physical structure. A TaN layer is deposited on a $\mathrm{SiO}_{2}$ layer of thickness $1.2 \mu \mathrm{m}$. TaN is used because of the remarkable stability of its electrical characteristics with temperature (Thermal Coefficient Resistor of the order of \pm 100 p.p.m. $/{ }^{\circ} \mathrm{C}$ ). The silicon substrate is $\mathrm{p}+$ doped and presents a bulk resistivity from 18 up to $22 \mathrm{~m} \Omega . \mathrm{cm}$.

Branches $Z_{1}$ and $Z_{2}$ model the electromagnetic coupling to the substrate through the $\mathrm{SiO}_{2}$ insulation layer. The thick oxide layer can be considered as a perfect dielectric and is represented with crossover capacitances $C_{\mathrm{ox} 1}$ and $C_{\mathrm{ox} 2}$. The substrate behavior depends upon 
both its resistivity and the frequency of the propagating wave. However, the substrate tends to behave as a lossy dielectric as the operating frequency enters the giga-hertz range. $C_{\text {sub1 }}$ and $C_{\text {sub2 }}$ reflect the dielectric nature of the substrate whereas $R_{\text {sub1 }}$ and $R_{\text {sub2 }}$, connected in parallel, represent the effective resistances due to ohmic losses in the substrate.

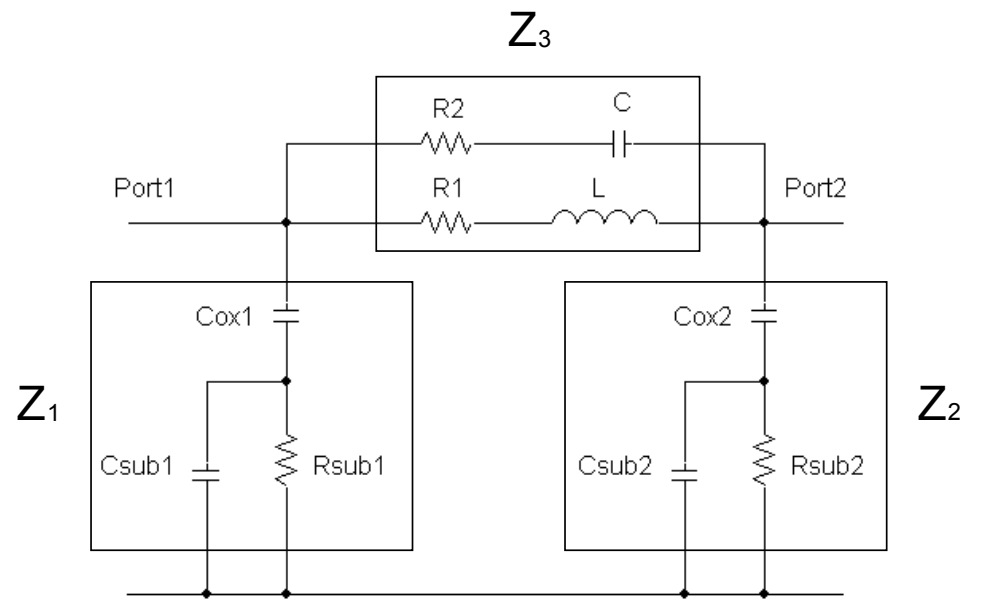

Figure 3. Single $\pi$-model.

The impedance $Z_{3}$ models the TaN deposit. $L$ expresses the lumped inductance of the structure, which includes the self and mutual between different lines, and can be evaluated using the Greenhouse algorithm (1974). Rigorously, the inductance value decreases with frequency seeing that the magnetic field penetration in the conductor diminishes with frequency. This effect remains slight and can be neglected because the inductance is principally determined by the external magnetic field distribution. The $R_{1}$ and $R_{2}$ resistances, controlling the $Z_{3}$ module respectively at low and high frequencies, approximate the frequency evolution of the current distribution in a conductor of finite thickness, this without introducing frequency dependent components. Lastly, the capacitance $C$ globally describes the proximity electric coupling existing between parallel conducting strips and the current crowding at the corners of rectangular turns.

\section{Model extraction}

Optimization is encompassed in a branch of mathematics named operations research, described by Bronson as being "concerned with the efficient allocation of scarce resources" (Bronson,1982). It is practically assimilated to curve fitting, defined as a procedure for finding the best fitting curve to a given set of points by minimizing the offsets of the points from the curve. The Figure-of-merit function, that measures the agreement between the data and the model, is conventionally arranged so that small values represent close agreement.

An efficient and accurate solution to any optimization problem is not only dependent on the size of the problem in terms of the number of adjustable variables, but also on characteristics of the objective functions. Optimization problems can be broadly categorized in three classes: linear problems, quadratic problems and lastly, the more difficult to solve, non-linear problems in which the objective functions and constraints are non-linear functions of the variables. A wide spectrum of numerical methods exists for finding function stationary values in a multi-dimensional topography, and can be separated in terms of the derivative information that is, or is not used. For instance, the downhill simplex search, due to Nelder and Mead (Nelder and Mead,1965), requires only function evaluations, not derivatives, whereas gradient based techniques such as the Levenberg-Marquardt method (More, 1977) use information about the slope of the function to dictate a direction of search where the minimum is thought to lie. 


\section{EXPERIMENTAL MODELING OF MONOLITHIC RESISTORS}

\subsection{Guess-timate issue}

An optimization process can take many iterations to converge and can be sensitive to numerical problems such as truncation and round-off error in gradient calculations. Thus, most optimization problems benefit from good starting guesses and it appears that the initial value aptness is often the cornerstone of a successful optimization. This point is all the most critical that the objective responses reveal a strong sensitivity to the different parameters. Indeed, the associated error functions are then uneven and present steep slopes, which can hold even the most subtle optimization algorithms in check. The aim of the mathematical relations introduced in this paper is to reduce the system degrees of freedom and provide the trial function parameters with relevant initial values. From this estimate, it is then possible to closely match the measured data by the refinement of these parameters. This approach is simple to implement with most commercial simulators that have the capability to optimize circuit parameters, and it requires little computation time to refine the small of parameters in a decoupled lumped-element $\pi$-model.

In order to gain clarity, the scope of our demonstration is limited to the elementary cell $Z_{3}$. The developed method rests on a thorough analysis of the function real part $\mathfrak{R}\left[Z_{3}(\omega)\right]$.

$$
\Re e\left[Z_{3}(\omega)\right]=\frac{R_{1}+\left(R_{1}+R_{2}\right) \cdot R_{1} R_{2} C^{2} \omega^{2}+R_{2} \cdot \frac{\omega^{4}}{\omega_{0}^{4}}}{\left[1-\frac{\omega^{2}}{\omega_{0}^{2}}\right]^{2}+\left(R_{1}+R_{2}\right)^{2} C^{2} \omega^{2}} \quad \text { where } \omega_{0}=\frac{1}{\sqrt{L C}}
$$

This function depends on one experimental variable: $\omega$, and four fitting parameters: $R_{1}, R_{2}, L$ and $C$. All the following results, summed up in Table 1, can be easily extended to the substrate coupling models $Z_{1}$ and $Z_{2}$, observing that the serial capacitance $C_{\mathrm{ox}}$ has no effect on the impedance real part.

\subsection{DC and AC resistances}

$R_{1}$ and $R_{2}$ values can theoretically be derived respectively from low and high frequency measurements, i.e.:

$$
\lim _{\omega \rightarrow 0} \mathfrak{R} e\left[Z_{3}(\omega)\right]=R_{1} \quad \lim _{\omega \rightarrow+\infty} \mathfrak{R} e\left[Z_{3}(\omega)\right]=R_{2}
$$

The approximations needed to establish statements (2) have been formalized to give rise to low and high frequency (LF and HF) conditions, see Table 1. These relations permit to quantitatively evaluate the veracity of the implicit assumption that the highest frequency sample measured can be assimilated to infinity and the lowest frequency sample to zero. If the low frequency resistance $R_{1}$ can always be evaluated with a DC measurement, the determination of $R_{2}$ as a limit is trickier. The impedance imaginary part $\Im m\left[Z_{3}(\omega)\right]$ is being studied at present in order to avoid element determination from limit conditions.

\subsection{Inductance and capacitance}

$$
\begin{array}{lll}
\text { Either } & \omega_{+}^{2}=\frac{L-l_{1}}{L-l_{2}} \cdot \omega_{0}^{2}, & \omega_{-}^{2}=-\frac{R_{1}}{R_{2}} \cdot \omega_{0}^{2}, \\
\text { with } & l_{1}=\frac{R_{1} C}{2}\left(R_{1}+R_{2}\right), & l_{2}=\frac{R_{2} C}{2}\left(R_{1}+R_{2}\right) .
\end{array}
$$

The first-order derivative with respect to the pulsation $\omega$ is the following (3): 


$$
\frac{d}{d \omega}\left\{\mathfrak{R}\left[Z_{3}(\omega)\right]\right\}=\frac{2 \omega R_{2} \cdot\left[R_{2}\left(R_{1}+R_{2}\right) C^{2}-\frac{2}{\omega_{0}^{2}}\right]\left(\omega^{2}-\omega_{+}^{2}\right)\left(\omega^{2}-\omega_{-}^{2}\right)}{\omega_{0}^{4} \cdot\left\{\left[1-\frac{\omega^{2}}{\omega_{0}^{2}}\right]^{2}+\left(R_{1}+R_{2}\right)^{2} C^{2} \omega^{2}\right\}^{2}}
$$

Analysis of this function leads to trinomials and fourth degree polynomials. Basically, all the relations concerning $L$ and $C$ are results of the discriminant positivity criterion, in order to ensure real and positive solutions. Three different cases were distinguished depending on the relation between $R_{1}$ and $R_{2}$. For each case, the existence condition of an extremum transferred to inductance $L$, the additive restricting relations determining the extremum nature, the capacitance intervals and the correlated $L_{1}$ and $L_{2}$ values compatible with an extremum at pulsation $\omega_{1}$, are derived (see Table 1). It must be pointed out that the system has, in the worst, case two remaining degrees of freedom $\left(R_{2}\right.$ and $\left.C\right)$, and in most cases one degree of freedom $(C)$.

Table 1: $Z_{3}$ elementary cell "cartography".

\begin{tabular}{|c|c|c|c|c|}
\hline$R$ relation & $R_{1}<R_{2}$ & $R_{1}=R_{2}$ & \multicolumn{2}{|c|}{$R_{1}>R_{2}$} \\
\hline $\begin{array}{l}\text { Limit inductances } \\
l_{1}, l_{2}\end{array}$ & $l_{1}=\frac{R_{1} C}{2}\left(R_{1}+R_{2}\right) \quad l_{2}=\frac{R_{2} C}{2}\left(R_{1}+R_{2}\right)$ & $l=R_{1}^{2} C$ & $l_{1}=\frac{R_{1} C}{2}\left(R_{1}+R_{2}\right)$ & $l_{2}=\frac{R_{2} C}{2}\left(R_{1}+R_{2}\right)$ \\
\hline $\begin{array}{c}\text { Extremum } \\
\text { existence intervals }\end{array}$ & $L \in] 0, l_{1}[\cup] l_{2},+\infty[$ & $L \in] 0, l[\cup] l,+\infty[$ & \multicolumn{2}{|c|}{$L \in] 0, l_{2}[\cup] l_{1},+\infty[$} \\
\hline \multirow{2}{*}{ Extremum nature } & $L \in] l_{2},+\infty[$ & $L \in] l,+\infty[$ & $L \in] 0, l_{2}[$ & $L \in] l_{1},+\infty[$ \\
\hline & maximum & maximum & mіпітит & maximum \\
\hline $\begin{array}{l}\text { Limit resistance } \\
\quad R_{\text {lim }}\end{array}$ & $\varnothing$ & $\varnothing$ & $2 R_{1} \cdot \frac{\sqrt{1} R_{1}}{2-\sqrt{1-\frac{R_{2}}{R_{1}}}}$ & $2 R_{1} \cdot \frac{1+\sqrt{1-\frac{R_{2}}{R_{1}}}}{2+\sqrt{1-\frac{R_{2}}{R_{1}}}}$ \\
\hline $\begin{array}{l}\text { Inductance giving } \\
\text { a resonance at } \\
\text { pulsation } \omega_{1}\end{array}$ & $L_{1,2}=\frac{1}{2 \omega_{1}^{2} C}+\frac{l_{2}}{2} \cdot\left[1 \pm \sqrt{\left(1-\frac{C_{1}^{2}}{C^{2}}\right) \cdot\left(1-\frac{C_{2}^{2}}{C^{2}}\right)}\right]$ & $L=\frac{1}{\omega_{1}^{2} C}$ & \multicolumn{2}{|c|}{$L_{1,2}=\frac{1}{2 \omega_{1}^{2} C}+\frac{l_{2}}{2} \cdot\left[1 \pm \sqrt{\left(1-\frac{C_{1}^{2}}{C^{2}}\right) \cdot\left(1-\frac{C_{2}^{2}}{C^{2}}\right)}\right.$} \\
\hline $\begin{array}{l}L-C \text { compatibility } \\
\text { condition }\end{array}$ & $\varnothing$ & $\varnothing$ & \multicolumn{2}{|c|}{$\left.C \in] 0, C_{2}\right] \cup\left[C_{1},+\infty[\right.$} \\
\hline$C_{1}$ & $\frac{1}{\omega_{1} R_{2}} \sqrt{\frac{2}{1+\frac{R_{2}}{R_{1}}}} \cdot\left[1+i \sqrt{\frac{R_{2}}{R_{1}}}-1\right]$ & $\frac{1}{\omega_{1} R_{2}}$ & \multicolumn{2}{|c|}{$\frac{\overline{R_{2}}}{\frac{R_{2}}{R_{2}}} \cdot\left[1-\frac{R_{2}}{2 R_{1}}+\sqrt{1-\frac{R_{2}}{R_{1}}}\right]^{\frac{1}{2}}$} \\
\hline$C_{2}$ & $\frac{1}{\omega_{1} R_{2}} \sqrt{\frac{2}{1+\frac{R_{2}}{R_{1}}}} \cdot\left[1-i \sqrt{\frac{R_{2}}{R_{1}}-1}\right]$ & $\frac{1}{\omega_{1} R_{2}}$ & $\frac{2}{\omega_{1} R_{2} \sqrt{1+\frac{R_{2}}{R_{1}}}} \cdot 1$ & $\left.\frac{R_{2}}{2 R_{1}}-\sqrt{1-\frac{R_{2}}{R_{1}}}\right]^{\frac{1}{2}}$ \\
\hline \multirow{2}{*}{$\begin{array}{l}\text { Bracketing } \\
\text { intervals } \\
L_{1}, L_{2}\end{array}$} & \multirow{2}{*}{$\left.L_{1} \in\right] l_{2},+\infty[$} & \multirow{2}{*}{$L \in] 0, l[\cup] l,+\infty[$} & $\left.C \in] 0, C_{2}\right]$ & $C \in\left[C_{1},+\infty[\right.$ \\
\hline & & & $\left.\left(L_{1}, L_{2}\right) \in\right] l_{1},+\infty\left[^{2}\right.$ & $\left.\left(L_{1}, L_{2}\right) \in\right] 0, l_{2}\left[^{2}\right.$ \\
\hline \multirow{2}{*}{$\begin{array}{l}\text { Inductance choice } \\
\text { criterion }\end{array}$} & maximum & \multirow{2}{*}{$\varnothing$} & $\Re e\left[Z_{3}\left(\omega_{1}\right)\right]<R_{\lim }$ & $\mathfrak{R e}\left[Z_{3}\left(\omega_{1}\right)\right]>R_{\text {lim }}$ \\
\hline & $L=L_{2}$ & & $L=L_{2}$ & $L=L_{1}$ \\
\hline $\begin{array}{l}\text { LF hypothesis } \\
\text { validity }\end{array}$ & $\omega_{\min }<<\frac{1}{\left(R_{1}+R_{2}\right) C}$ & \multicolumn{3}{|c|}{$\omega_{\min }<<\sqrt{\frac{R_{1}}{\left(L+R_{1} R_{2} C\right)\left(R_{1}+R_{2}\right) C}}$} \\
\hline $\begin{array}{l}\text { HF hypothesis } \\
\text { validity }\end{array}$ & $\omega_{\max }>>\frac{1}{\sqrt{L C}}$ & $\omega_{\max } \gg \frac{R_{1}+R_{2}}{L}$ & $\omega_{\max }>\sqrt{\frac{R_{1}}{R_{2} L C}}$ & $\omega_{\max } \gg \frac{R_{1}+R_{2}}{L}$ \\
\hline
\end{tabular}




\section{EXPERIMENTAL MODELING OF MONOLITHIC RESISTORS}

\section{Examples and discussions}

The $R_{1}, R_{2}, L$ and $C$ parameters of the compact model have been shown to be identifiable through a combination of parameter identification and fitting with the aid of a computer-driven optimizer. In our study the ELDO's general-purpose electrical circuit optimization program Opsim is used, relying on a traditional gradient descent method. To demonstrate the guesstimate accuracy, the extraction procedure outlined in the previous part is applied to the case of a $1 \mathrm{k} \Omega$ integrated resistor.

- The DC resistance $R_{1}$ is simply derived from the corresponding limit relation (2). $R_{1}=\mathfrak{R e}\left[Z_{3}\left(\omega_{\min }\right)\right]=975 \Omega$.

- The profile $\mathfrak{R} e\left[Z_{3}(\omega)\right]$ is not constant in the high frequency domain. Hence, the precision on the AC resistance determination is limited to a domain centered on $\mathfrak{R} e\left[Z_{3}\left(\omega_{\max }\right)\right]=965 \Omega$, for instance $[930,1000] \Omega$. The parameter initial value is set to the interval mean value, $R_{2}=965 \Omega$.

- Measurements show a self-resonant frequency at approximately $0.5 \mathrm{GHz}$ that is to say $\omega_{1}=$ $3.15 \times 10^{9}$ rad.s ${ }^{-1}$. Assuming $R_{1}>R_{2}$, one can conclude that $L$ has to belong to the interval ]$l_{1},+\infty[$ and $C$ to the interval $\left.] 0, C_{2}\right]$ in order to be consistent with the maximum observed. The value of the limit capacitance $C_{2}$ is straightforwardly deduced from $R_{1}$ and $R_{2}$ terms: $C_{2} \cong 0.3 \mathrm{pF}$, from which we infer the following capacitance variation domain $C \in[0.15,0.35] \mathrm{pF}$. Figure 4 represents the compatible inductance solutions $L_{1}$ and $L_{2}$ as functions of the capacitance $C$.

- We have $R e\left[Z_{3}\left(\omega_{1}\right)\right]=1052 \Omega$ and $R_{\lim }=1022 \Omega$. The inequality $\mathfrak{R} e\left[Z_{3}\left(\omega_{1}\right)\right]>R_{\lim }$ is verified, which enforces the choice of the solution $L_{1}$. The inductance interval correlated to the capacitance range is consequently deduced: $L \in[300,700] \mathrm{nH}$.

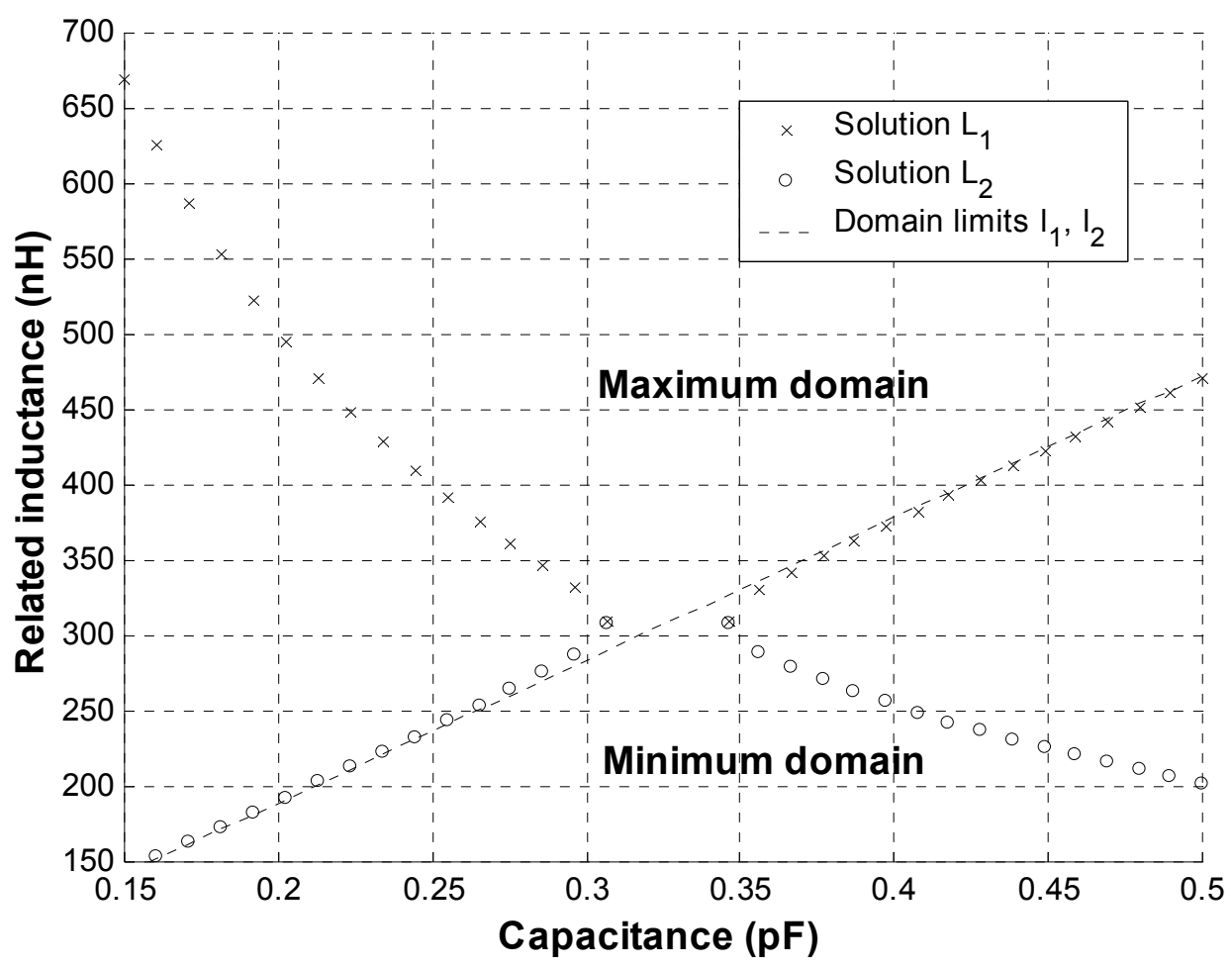

Figure 4. Compatible $L$ and $C$ values. 


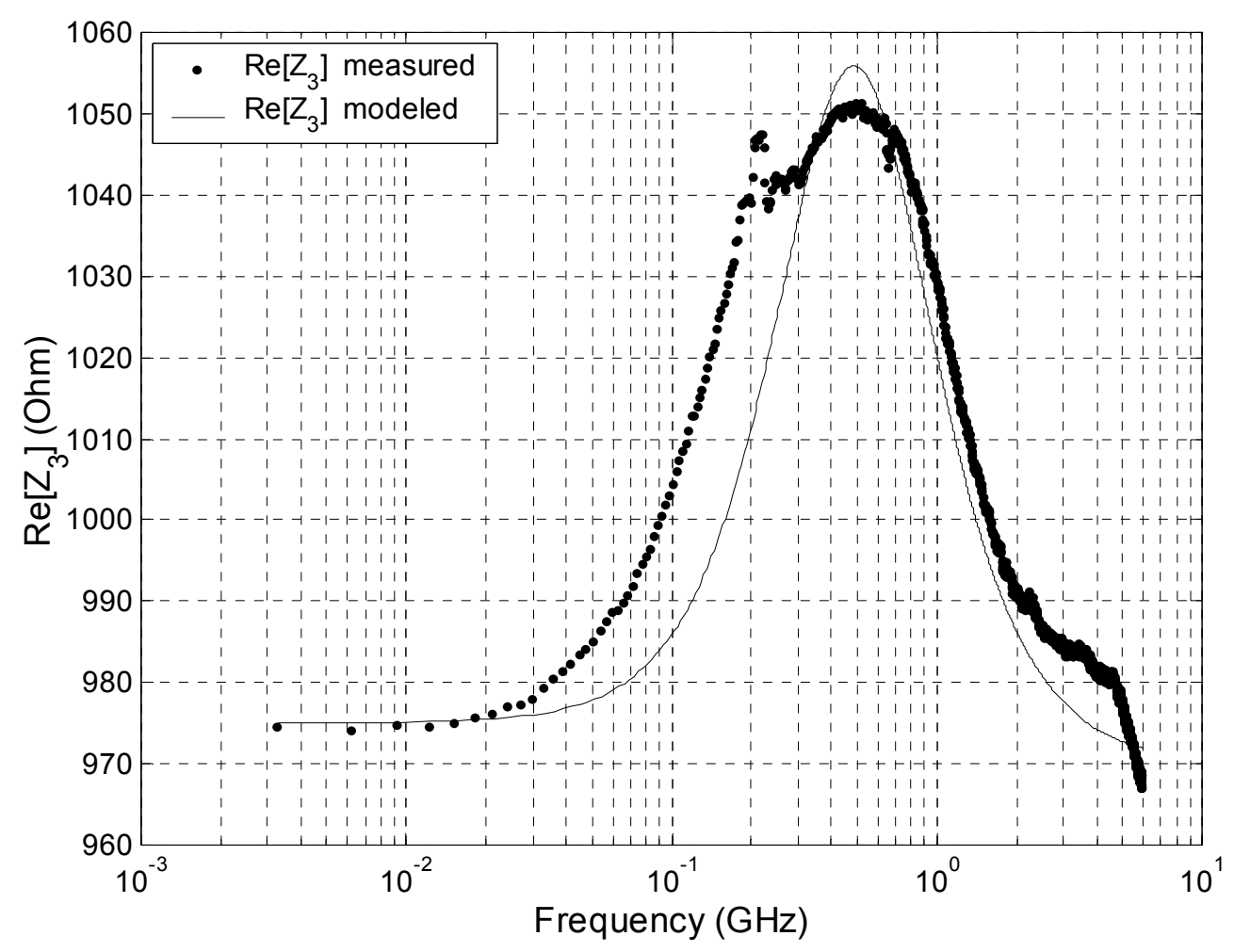

Figure 5. $1 \mathrm{k} \Omega \mathrm{TaN}$ resistor, real part.

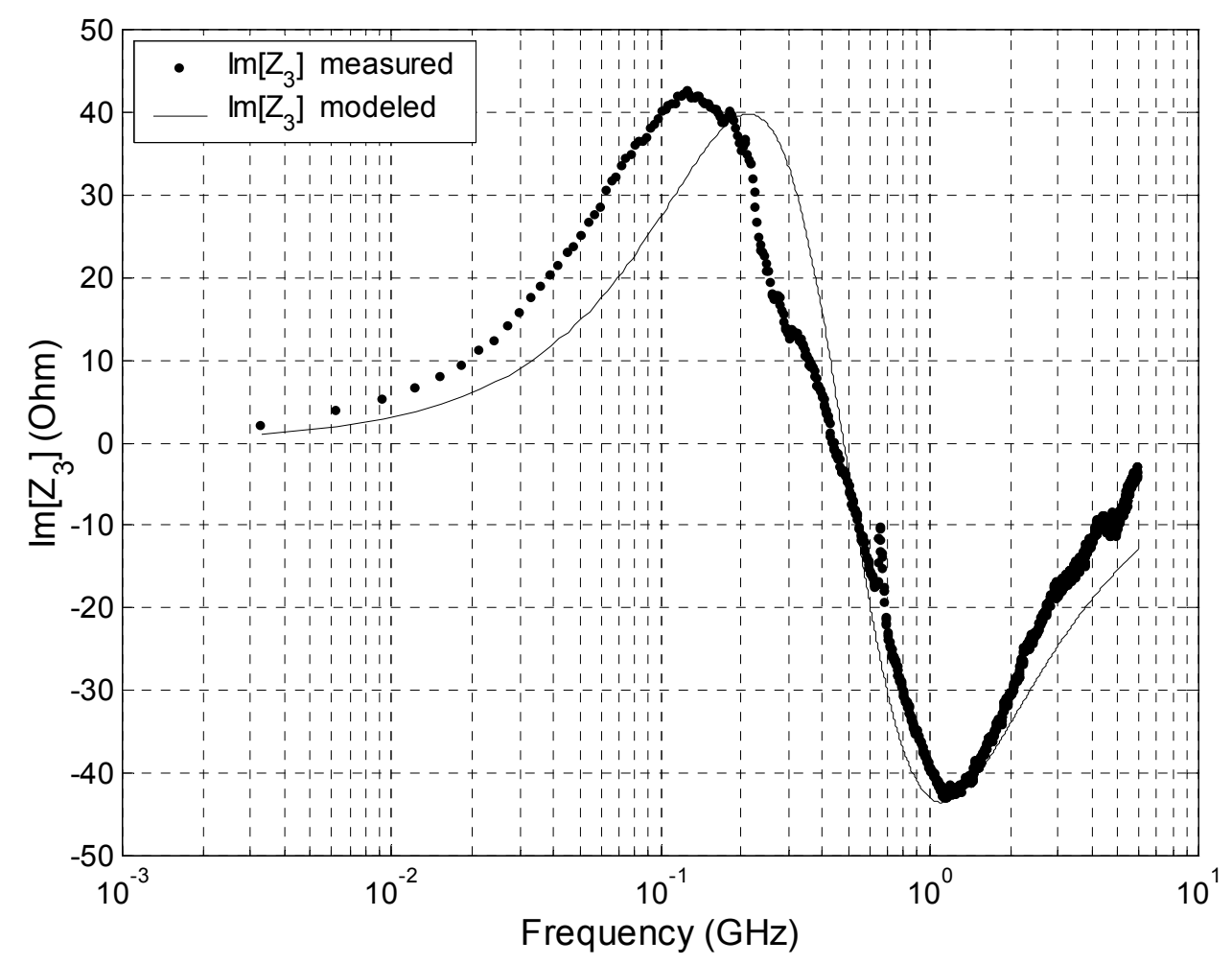

Figure 6. $1 \mathrm{k} \Omega$ TaN resistor, imaginary part. 


\section{EXPERIMENTAL MODELING OF MONOLITHIC RESISTORS}

Measured and modeled values of $Z_{3}$ are plotted as in Figure 5 and Figure 6 in semilogarithmic scale. The optimization stretches from $3 \mathrm{MHz}$ to $6 \mathrm{GHz}$ and turns on both the real and imaginary parts of the impedance as objective functions. The results are the following: $R_{2}=972 \Omega, L=339 \mathrm{nH}$ and $C=0.31 \mathrm{pF}$. A correct agreement is noticed between the real and imaginary parts of the impedance measured and the lumped element model. The coherence of the established model is controlled evaluating the low and high frequency requirements concerning $\omega_{\min }=2.07 \times 10^{7} \mathrm{rad} . \mathrm{s}^{-1}$ and $\omega_{\max }=3.77 \times 10^{10} \mathrm{rad} . \mathrm{s}^{-1}$. The first condition imposes $\omega_{\min }<<1.60 \times 10^{9} \mathrm{rad} . \mathrm{s}^{-1}$, and the second leads to $\omega_{\max }>>5.74 \times 10^{9} \mathrm{rad} . \mathrm{s}^{-1}$. The latter is not totally fulfilled and consequently, it is confirmed that the infinite limit does not appear in this case as the best way to determine the constant $R_{2}$.

The extracted value of the inductance, $L=339 \mathrm{nH}$, is much too large to represent a physical reality. In order to evaluate the substrate nature influence on the structural modeling of the deposit $\left(Z_{3}\right)$, a component with the same geometry has been processed on a glassy substrate. It appears that the resonance is much less pronounced and can be neglected. Indeed, as a dielectric material, glass presents a very weak conductivity, which considerably limits any magnetic coupling. We can therefore conclude that impedance $Z_{3}$ cannot be fully assimilated to the TaN deposit on the oxide surface, but also reflects a coupling with the silicon substrate. This fact is simply explained if we keep in mind that this interaction is intrinsically distributed and cannot be rigorously reported on two parallel branches $\left(Z_{1}, Z_{2}\right)$. Moreover, applying the foregoing procedure to resistors of different layouts, it has been proved that while the size of the resistor becomes bigger and bigger, the different coupling effects will be more and more complicated, and it becomes harder and harder to fit the measurement due to the simplification inherent to a one-cell model.

\section{Conclusions}

This study has pointed out optimization issues inherent to the over-sensitivity of the measured responses to the different fitting variables. In order to solve this problem, an analytical direct method for circuit elements extraction has been developed. Decoupling substrate and component modeling through a $\pi$-representation of a two-port network limits the number of fitting variables to determine simultaneously what makes parameter extraction by an optimization process more stable. If the results, qualitatively correct, are encouraging, it is clear that a single $\pi$-type lumped-element circuit is inadequate to correctly describe components of complex geometries over the total frequency domain. Our research efforts are now focusing on extending the extraction procedure to a multi-cell model.

\section{References}

BRONSON, R., 1982. Schaum's outline of theory and problems of operations research. New York, McGraw-Hill.

CHEN, D.X., and MUNOZ, J.L., 1997. Theoretical eddy-current permeability spectra of slabs with bar domains. IEEE Transactions on Magnetics, 33: 2229-2244.

CHOI, K.L., and SWAMINATHAN, M., 1999. Synthesis of equivalent circuits for two-port integral passive components. Conference on High Density Packaging and MCMs.

CIAMPOLINI, P. 1996. Integrated FDTD and solid-state device simulation. IEEE Microwave and Guided Wave Letters, 6: 419-421.

GREENHOUSE, H., 1974. Design of planar rectangular microelectronic inductor. IEEE Transactions PHP, 10: 101-109.

HARRINGTON, R.F., 1968. Field computation by moment methods. The Macmillan Co., New York.

ITOH, T., 1989. Numerical techniques for microwave and millimeter-wave passive structure. New York, John Wiley \& sons. 
JOHNS, P.B., 1987. A symmetrical condensed node for the TLM method. IEEE Transactions on Microwave Theory Techniques, 35: 370-377.

KOLDING, T.E., 1999. On-wafer calibration techniques for giga-hertz CMOS measurements. Proceeding IEEE 1999 International Conference on Microelectronic Test Structures, 12: 105110.

LONG, J.R., and COPELAND, M.A., 1997. The modeling, characterization, and design of monolithic inductors for silicon RF IC's. IEEE Journal of Solid-State Circuits, 32: 357-369.

MORE, J.J., 1977. The Levenberg-Marquardt algorithm : implementation and theory. Numerical Analysis, Lecture Notes in Mathematics 630, ed. G.A. Watson, Springer Verlag, 105-116.

NELDER, J.A., and MEAD, R., 1965. A simplex method for function minimization. Computer Journal, 7: 308-313.

YUE, C.P., and WONG, S.S., 2000. Physical modeling of spiral inductors on silicon. IEEE Transactions on Electron Devices, 47: 560-568.

ZHAO, J., FRYE, R.C., DAI, W.W., and TAI, K.L., 1997. S parameter-based experimental modeling of high $\mathrm{Q}$ MCM inductor with exponential gradient learning algorithm. IEEE Transactions on Components, Packaging, and manufacturing Technology - Part B, 20: 202210.

Received 11 June 2001

Accepted 10 February 2002 